\title{
AAV-DC-CTL Tumor Cell Targeting Therapy
}

National Cancer Institute

\section{Source}

National Cancer Institute. AAV-DC-CTL Tumor Cell Targeting Therapy. NCI Thesaurus.

Code $C 107677$.

A tumor-targeted therapy consisting of cytotoxic T lymphocytes (CT Ls) that are exposed ex vivo to dendritic cells (DCs) transfected with a recombinant adeno-associated virus ( $\mathrm{rAAV}$ ) encoding a specific tumor associated antigen (TAA) gene. Autologous peripheral blood mononuclear cells (PBMC) are collected and separated into monocytes and lymphocytes. The monocytes are infected with the rAAV that carries the TAA gene; this is followed by co-culture of the monocyte-derived, mature rAAV-transfected DCs and the isolated lymphocytes to generate CT Ls against the specific TAA. These CT Ls are transfused back into the patient for cancer therapy. The CT Ls specifically kill tumor cells expressing the TAA, which results in a reduction of tumor cell proliferation. 